\title{
Multiple bosonic mode coupling in the charge dynamics of the electron-doped superconductor $\left(\mathrm{Pr}_{2-x} \mathrm{Ce}_{x}\right) \mathrm{CuO}_{4}$
}

\author{
E. Schachinger, ${ }^{1, \text { * }}$ C. C. Homes, ${ }^{2}$ R. P. S. M. Lobo, ${ }^{3}$ and J. P. Carbotte ${ }^{4,5}$ \\ ${ }^{1}$ Institute of Theoretical and Computational Physics, \\ Graz University of Technology, A-8010 Graz, Austria \\ ${ }^{2}$ Condensed Matter Physics and Materials Science Department, \\ Brookhaven National Laboratory, Upton, NY 11973, USA \\ ${ }^{3}$ Laboratoire Photons et Matière (CNRS UPR 5), LPS-ESPCI, \\ Université Pierre et Marie Curie, 10 rue Vauquelin, F-75231 Paris Cedex 5, France \\ ${ }^{4}$ Department of Physics and Astronomy, McMaster University, Hamitlon, Ontario N1G 2W1, Canada \\ ${ }^{5}$ The Canadian Institute for Advanced Research, Toronto, Ontario M5G 1Z8, Canada
}

(Dated: November 26, 2018)

\begin{abstract}
We analyze optical spectroscopy data of the electron-doped superconductor $\left(\operatorname{Pr}_{2-x} \mathrm{Ce}_{x}\right) \mathrm{CuO}_{4}$ (PCCO) to investigate the coupling of the charge carriers to bosonic modes. The method of analysis is the inversion of the optical scattering rate $\tau_{\mathrm{op}}^{-1}(\omega, T)$ at different temperatures $T$ by means of maximum entropy technique combined with Eliashberg theory. We find that in the superconducting state the charge carriers couple to two dominant modes one at $\sim 12 \mathrm{meV}$ and a second one at $\sim 45 \mathrm{meV}$ as well as to a high energy background. The low energy mode shows a strong temperature dependence and disappears at or slightly above the critical temperature $T_{c}$. The high energy mode exists above $T_{c}$ and moves towards higher energies with increasing temperatures. It becomes less prominent at temperatures $>100 \mathrm{~K}$ above which it evolves into a typical spin-fluctuation background. In contrast to the hole-doped High- $T_{c}$ superconductors PCCO proves to be a superconductor close to the dirty limit.
\end{abstract}

PACS numbers: 74.20.Mn 74.25.Gz 74.72.-h

\section{INTRODUCTION}

The optical scattering rate $\tau_{\text {op }}^{-1}(\omega)$ in the infrared regime evolved into an important tool to extract microscopic information on the coupling of charge carriers to bosonic modes in the form of the electron-exchange boson interaction spectral density $I^{2} \chi(\omega)$ in the High- $T_{c}$ cuprates. The definition of the optical scattering rate itself is based on a generalized Drude form valid for correlated electron systems:

$$
\sigma(\omega, T)=\frac{i \Omega_{p}^{2}}{4 \pi} \frac{1}{\omega-2 \Sigma_{\mathrm{op}}(\omega, T)} .
$$

This equation relates the complex optical self energy $\Sigma_{\text {op }}(\omega, T)$ at a given temperature $T$ to the complex optical conductivity $\sigma(\omega, T)$. The optical scattering rate is related to the imaginary part of the optical self energy $\Sigma_{2, \mathrm{op}}(\omega, T)$ via

$$
\tau_{\text {op }}^{-1}(\omega, T)=-2 \Sigma_{2, \mathrm{op}}(\omega, T)=\frac{\Omega_{p}^{2}}{4 \pi} \Re \mathrm{e}\left\{\sigma^{-1}(\omega, T)\right\} .
$$

Here $\Omega_{p}$ is the plasma frequency and $\omega$ an energy variable.

It was first demonstrated by Marsiglio et al $\frac{1}{\underline{1}}$ that there exists an approximate relation between $I^{2} \chi(\omega)$ and $\tau_{\mathrm{op}}^{-1}(\omega)$ of the form:

$$
I^{2} \chi(\omega) \simeq W(\omega)=\frac{1}{2 \pi} \frac{d^{2}}{d \omega^{2}}\left[\frac{\omega}{\tau_{\mathrm{op}}(\omega)}\right] .
$$

This relation is valid only at low temperatures and up to energies at which $W(\omega)$ first becomes negative, i.e. unphysical. This second derivative method was used by Carbotte et $a l^{2} \underline{2}$ to demonstrate that in the optimally doped system $\mathrm{YBa}_{2} \mathrm{Cu}_{3} \mathrm{O}_{6+\delta}$ (YBCO) the quasiparticles couple to a boson resonance at $41 \mathrm{meV}$ which corresponds to a spin one resonance observed by neutron scattering in the imaginary part of the spin susceptibility. ${ }^{3,4}$ Moreover, the temperature dependence of this resonance peak was found to be identical to the $T$ variation of the equivalent structure in the spectral function $I^{2} \chi(\omega)$ derived

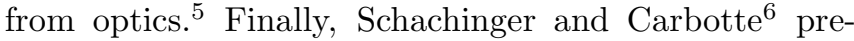
dicted such a spin one resonance to exist in the thallium compound $\mathrm{Tl}_{2} \mathrm{Ba}_{2} \mathrm{CuO}_{6+\delta}(\mathrm{Tl} 2201)$. This was later con-

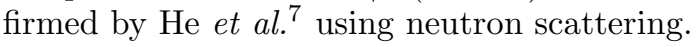

The next step in the development of methods which help to extract information on the electron-exchange boson interaction spectral density $I^{2} \chi(\omega)$ from optics, was made by Dordevic et $a l^{.} \underline{8}$ who developed a new method based on inverse theory. These authors concentrated on the approximate relation

$$
\tau_{\mathrm{op}}^{-1}(\omega, T)=\tau_{\mathrm{imp}}^{-1}+\int_{0}^{\infty} d \nu K(\omega, \nu ; T) I^{2} \chi(\nu)
$$

reported by Shulga et al. $\frac{9}{\mathrm{Eq}}$. (44) is based on Eliashberg theory and is valid in the normal state. Here $\tau_{\text {imp }}^{-1}$ is an energy independent impurity scattering rate and the kernel $K(\omega, \nu ; T)$ is given by:

$$
\begin{aligned}
K(\omega, \nu ; T)= & \frac{\pi}{\omega}\left[2 \omega \operatorname{coth}\left(\frac{\nu}{2 T}\right)-(\omega+\nu) \operatorname{coth}\left(\frac{\omega+\nu}{2 T}\right)\right. \\
& \left.+(\omega-\nu) \operatorname{coth}\left(\frac{\omega-\nu}{2 T}\right)\right] .
\end{aligned}
$$


Relation (41) was extended by Carbotte and Schachinger 10 to the superconducting state of a $d$-wave superconductor at $T=0$ using the kernel

$$
\begin{aligned}
K(\omega, \nu ; T=0)= & \frac{2 \pi}{\omega}\langle(\omega-\nu) \theta[\omega-2 \Delta(\vartheta)-\nu] \\
& \left.\times E\left(\sqrt{1-\frac{4 \Delta^{2}(\vartheta)}{(\omega-\nu)^{2}}}\right)\right\rangle_{\vartheta} .
\end{aligned}
$$

This kernel is based on a clean limit, i.e.: $\tau_{\mathrm{imp}}^{-1}=0$, perturbation theory expansion of BCS theory reported by P.B. Allen 11 for an $s$-wave superconductor which has also been considered by Dordevic et al. $\underline{\underline{8}}$ In as much as one can think of a $d$-wave superconductor as a superposition of $s$-wave with variable gaps, Eq. (6) follows as a first approximate generalization of Allen's work to $d$-wave. Here $\langle\cdots\rangle_{\vartheta}$ denotes the $\vartheta$-average which can be limited to the interval $\vartheta \in[0, \pi / 4]$ for symmetry reasons. Furthermore, $\Delta(\vartheta)=\Delta \cos (2 \vartheta)$ reflecting the $d$-wave symmetry of the superconducting order parameter. Eq. (6) ensures that the optical scattering rate is finite in the superconducting state for $\omega>0$. Finally, $E(x)$ is the complete elliptic integral of second kind and $\theta(x)$ is the step function.

Eq. (6) explains why in the superconducting state of a $d$-wave superconductor the signatures of the $I^{2} \chi(\nu)$ spectral density in the optical scattering rate $\tau_{\mathrm{op}}^{-1}(\omega)$ are displaced at least by the gap amplitude $\Delta, \underline{12,13,14}$ This is in contrast to a classical $s$-wave superconductor in which those signatures are displaced by $2 \Delta_{0}$, with $\Delta_{0}$ the gapedge. Finally, we would like to point out that according to Eq. (44) non-zero contributions to the positive definite spectral density $I^{2} \chi(\nu)$, i.e.: non-zero coupling of charge carriers to an exchange boson at energy $\nu$, will always result in an increase of the optical scattering rate because both kernels, Eqs. (5) and (6), are positive definite. Thus, depressions or pronounced peaks in the optical scattering rate $\tau_{\text {op }}^{-1}(\omega)$ cannot be explained by electron-exchange boson interaction and indicate additional influences like, for instance, infrared activated phonons or quasiparticle density of states effects.

The spectra derived from the deconvolution of Eq. (4) still contained negative and, thus, unphysical parts. Nevertheless, it was demonstrated by Dordevic et al. $\underline{\underline{8}}$ that the application of a deterministic constraint can result in the regularization of the problem which allows to the removal of unphysical negative values from the inverted spectral function $I^{2} \chi(\nu)$. The authors also demonstrated that such a regularization can result in a reduced quality of data reproduction.

In a final step, so far, in the development of inversion techniques it was demonstrated by Schachinger et al $\stackrel{15}{15}$ that the application of maximum entropy techniques 16 maximizing the generalized Shannon-Jaynes entropy 17

$$
S=\sum_{j=1}^{N}\left[a_{j}-m_{j}-a_{j} \log \frac{a_{j}}{m_{j}}\right]
$$

to deconvolute Eq. (4) will result in a positive definite spectral density $I^{2} \chi(\nu)$ ensuring best possible data reproduction. Here, the entropy $S$ measures the distance of a candidate vector $\mathbf{a}=\left\{a_{j} \mid j=1, \ldots, N\right\}$ from the default model vector $\mathbf{m}=\left\{m_{j} \mid j=1, \ldots, N\right\}$ which represents the most probable solution prior to the observation of any data. In case of insufficient background information it should be chosen constant, i.e.: $m_{j}=$ const, $\forall j$, as will be done throughout this paper.

To compensate for the approximate nature of Eq. (4) an additional least squares fit procedure based on Eliashberg equations which have been extended to $d$-wave superconductors (see Appendix ${ }_{\text {a }}$ ) proved, finally, to be very successful in the inversion of optical $\mathrm{Bi}_{2} \mathrm{Sr}_{2} \mathrm{CaCu}_{2} \mathrm{O}_{8+\delta}$ (Bi2212) data at various temperatures and doping levels 18

All this research resulted in one common denominator: in YBCO, Bi2212, and Tl2201 the optical data suggest coupling of the charge carriers to a pronounced boson mode, an 'optical' resonance, at energies which agree in most cases with the energies at which a spin one resonance is found by neutron scattering in the imaginary part of the spin susceptibility. In a new experiment Vignolle et al $\stackrel{19}{=}$ showed, using neutron scattering, that the imaginary part of the spin susceptibility in optimally doped $\left(\mathrm{La}_{2-x} \mathrm{Sr}_{x}\right) \mathrm{CuO}_{4}$ (LSCO) develops two peak structures, a resonance at the low energy of $12 \mathrm{meV}$ while the second peak was found at $\sim 50 \mathrm{meV}$. A maximum entropy analysis of optical data reported by Gao et al ${ }^{20}$ on epitaxial optimally doped LSCO thin films confirmed that, indeed, the electron-boson spectral density $I^{2} \chi(\omega)$ develops two peaks at the energies reported by neutron scattering. $\stackrel{21}{=}$ This is also in agreement with earlier results suggested by Zhou et al. ${ }^{22}$ from angular resolved photo emission spectroscopy (ARPES) experiments in underdoped samples. They interpreted their results as a coupling of the charge carriers to phonons which is in some contrast to the results reported by Vignolle et al. $\frac{19}{}$ and Gao et $a l^{20}$

Neutron scattering experiments by Wilson et al ${ }^{23}$ report the existence of a spin one resonance in the imaginary part of the spin susceptibility of $\left(\mathrm{Pr}_{1-x} \mathrm{LaCe}_{x}\right) \mathrm{CuO}_{4-\delta}$ (PLCCO) for $x=0.12$ with its peak at $\sim 11 \mathrm{meV}$. It is centered around $(\pi / 2, \pi / 2)$ in the two dimensional $\mathrm{CuO}$ Brillouin zone as is the case in all other High- $T_{c}$ superconductors and it disappears at temperatures above $T_{c}$. The doping $(x)$ dependence of this peak was studied by M. Fujita et al $\underline{24}$ Scanning tunneling microscope (STM) experiments performed on the same material by Niestemski et al.$^{25}$ support the existence of such a peak at $\sim 10 \mathrm{meV}$. As optical data are available in the similar system $\left(\mathrm{Pr}_{2-x} \mathrm{Ce}_{x}\right) \mathrm{CuO}_{4-\delta}(\mathrm{PCCO})$ we concentrate in this paper on the task to extract information on the electron-boson spectral density $I^{2} \chi(\omega)$ from optical scattering rates measured by Homes et al $l^{26}$ on a PCCO single crystal with $x=0.15\left(T_{c}=20 \mathrm{~K}\right)$ and from similar data reported by Zimmers et al $.27,28$ on thin, epitaxially grown PCCO films with $x=0.15\left(T_{c}=21 \mathrm{~K}\right)$. 
In Sec. II we discuss the samples used for the analysis in their main features. Data extraction techniques will also be discussed shortly within this section. Section III concentrates on the analysis of the optical data and the discussion of the important features of the $I^{2} \chi(\omega)$ spectra derived from optical data. Finally, Sec. IV gives a short summary. An appendix has been included to provide the necessary background information on the extended $d$-wave Eliashberg formalism used for impure systems.

\section{MATERIALS}

\section{A. The optimally doped $(x=0.15)$ PCCO single crystal}

We concentrate first on the experimental results reported by Homes et al $\underline{26}$ for an optimally doped PCCO single crystal $\left(x=0.15, T_{c} \sim 20 \mathrm{~K}\right)$. The authors report two plasma frequencies, the first value $\Omega_{p}=13000 \pm$ $200 \mathrm{~cm}^{-1}(1.64 \mathrm{eV})$ reflects only those carriers which participate in coherent "Drude" transport, and a larger value for $\Omega_{p}=19300 \mathrm{~cm}^{-1}(2.4 \mathrm{eV})$, which was determined from a modification of the finite-energy $f$-sum rule ${ }^{29.30}$ The two values of $\Omega_{p}$ obtained using these different approaches indicate that $\Omega_{p}$ is not well known in this material, and that its value depends heavily on the method chosen to extract it from the experiment. This leads to some uncertainty because according to Eq. (2) the plasma frequency sets the scale of the optical scattering rate. The smaller value of $\Omega_{p}$ finds its justification in a two-component system where the Drude response is not directly connected to the mid-infrared region which is conceived to be due to another band of electrons; this was the approach used in the original analysis of the PCCO single crystal data.$^{26}$ However, in this work we use a onecomponent approach and as a result the larger value of $\Omega_{p}$ is more appropriate. In this view Drude and infrared regions come from the same electrons with the infrared part coming from the incoherent boson-assisted processes, and the Drude originates from the coherent quasiparticle response part of the carrier spectral function. Following this argument, the experimental $\tau_{\mathrm{op}}^{-1}(\omega)$ data has been derived from the raw reflectance data using $\Omega_{p}=2.4 \mathrm{eV}$ together with the dielectric constant at infinity, $\epsilon_{\infty}$, set equal to four. It is worth remarking at this point that the dielectric function, $\epsilon(\omega)$, and the conductivity are related through the expression $\sigma(\omega)=-i \omega\left[\epsilon(\omega)-\epsilon_{\infty}\right] / 4 \pi$. Thus, $\epsilon_{\infty}$ is required to derive the imaginary part of the optical conductivity, and as a consequence the optical scattering rate is also influenced by this important parameter.

The optical scattering rate at the lowest normal state temperature reported $(30 \mathrm{~K})$ has a zero frequency offset of $\sim 22 \mathrm{meV}\left(\sim 177 \mathrm{~cm}^{-1}\right)$ which indicates substantial contributions from impurity scattering. In addition, there are sharp features in the scattering rate shown in Fig. 1(a) at approximately 306, 338, and $434 \mathrm{~cm}^{-1}$ $(\approx 37.9,41.9$, and $53.8 \mathrm{meV})$ which have been identi-

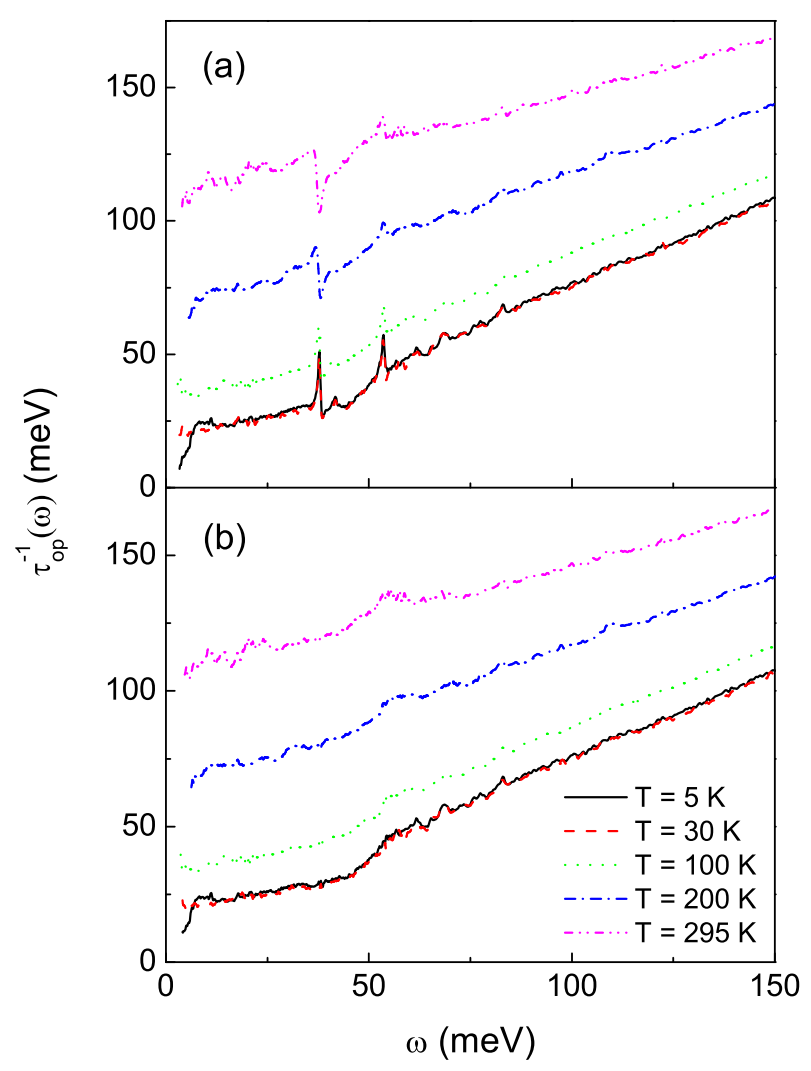

FIG. 1: (Color online) The optical scattering rate $\tau_{o p}^{-1}(\omega)$ in $\mathrm{meV}$ in an optimally-doped $(x=0.15)$ thin PCCO single crystal $\left(T_{c}=20 \mathrm{~K}\right)$ for several temperatures above and below $T_{c}$ with (a) the three infrared-active $E_{u}$ modes at $\approx 306,338$, and $434 \mathrm{~cm}^{-1}(\approx 37.9,41.9$, and $53.8 \mathrm{meV})$ present, and (b) with the same phonons removed.

fied as the infrared-active $E_{u}$ lattice vibrations in this material.26,31,32,33 In order to remove this sharp structure from the scattering rate, the dielectric function has been modeled using a series of Lorentz oscillators

$$
\epsilon(\omega)_{o s c}=\sum_{j} \frac{\omega_{p, j}^{2}}{\omega_{j}^{2}-\omega^{2}-i \gamma_{j} \omega},
$$

where $\omega_{j}, \gamma_{j}$ and $\omega_{p, j}$ are the frequency, width and effective plasma frequency of the $j$ 'th vibration, respectively. The oscillator parameters have been determined from fits to the real part of the optical conductivity using a simple linear background; the fitted oscillator values are then used to generate the phonon contribution to the complex dielectric function which is in turn subtracted from the experimentally determined values for the real and imaginary parts of the dielectric function, allowing the sharp phonon features to be removed from the optical scattering rate, as shown in Fig. 1(b). 


\section{B. The optimally doped $(x=0.15)$ PCCO thin film}

We also analyzed data published on thin films by Zimmers et $a l .27,28$ and concentrate on the $x=0.15$ sample. The reflectivity of a thin film also contains information on the optical properties of the underlying substrate. Hence one cannot obtain the film optical functions using a straight-forward Kramers-Kronig inversion. In the case of the PCCO films, the substrate response was determined separately. The reflectivity of the total (film plus substrate) system was then fitted by constructing a suitable Drude-Lorentz dielectric function for the film. ${ }^{34}$ One should note that the parameters used in this Drude-Lorentz dielectric function are not to be taken as representative of intrinsic excitations. They should be looked at as a convenient parameterization of the material dielectric function. Once this dielectric function is known, any other optical function can be generated straightforwardly.

Figure 2(a) presents the optical scattering rate $\tau_{\text {op }}^{-1}(\omega)$ vs $\omega$ for the optimally doped, epitaxially grown thin PCCO film $\left(x=0.15, T_{c}=21 \mathrm{~K}\right)$. The plasma frequency, calculated from a finite sum rule, is $\Omega_{p}=17570 \mathrm{~cm}^{-1}$ $(2.18 \mathrm{eV})$ and $\epsilon_{\infty}$ was set to four. Figure 2(b) shows the scattering rate once phonons have been eliminated.

The zero frequency offset of the optical scattering rate $\tau_{\mathrm{op}}^{-1}(0)=32.5 \mathrm{meV}\left(\sim 262 \mathrm{~cm}^{-1}\right)$ at $T=25 \mathrm{~K}$ which suggests a much higher impurity concentration in comparison to the single crystal discussed in the previous subsection. The overall shape of the scattering rate in the $x=0.15$ film is similar to the crystal response. The phonons in the film tend to be broader and less screened by free carriers than in the crystal. These effects are consistent with a larger disorder and smaller weight of the coherent peak in the film, as we describe below.

There is a scaling by roughly a factor of two required if one wants to compare directly the $x=0.15$ film and crystal data. It comes from the difference in the absolute value of their respective scattering rates. It is worth noticing that this is not an effect originating in the scattering rate of carriers participating in the coherent transport. As discussed in Sec. IA taking into account the coherent transport alone, the low frequency optical conductivity of the $x=0.15$ single crystal at $T=30 \mathrm{~K}$ can be described by a Drude peak having $\Omega_{p}=1.61 \mathrm{eV}\left(13000 \mathrm{~cm}^{-1}\right)$ and $\tau_{\mathrm{op}}^{-1}(\omega=0) \approx 11 \mathrm{meV}$ $\left(90 \mathrm{~cm}^{-1}\right)$. Performing the same analysis on the $x=0.15$ film at $T=25 \mathrm{~K}$ one obtains $\Omega_{p}=1.18 \mathrm{eV}\left(9500 \mathrm{~cm}^{-1}\right)$ and $\tau_{\mathrm{op}}^{-1}(\omega=0)=11 \mathrm{meV}$. The scattering rate is the same but the weight of the Drude peak in the film is significantly smaller. As the film and the crystal compositions are nominally the same, the $f$-sum rule states that the area under their respective optical conductivities should be the same. Hence, the weight lost in the coherent peak of the film is redistributed as an incoherent background. This effect will produce, as observed, a higher mid-infrared optical conductivity in the film and hence a broader incoherent scattering rate. Thus, a fac-

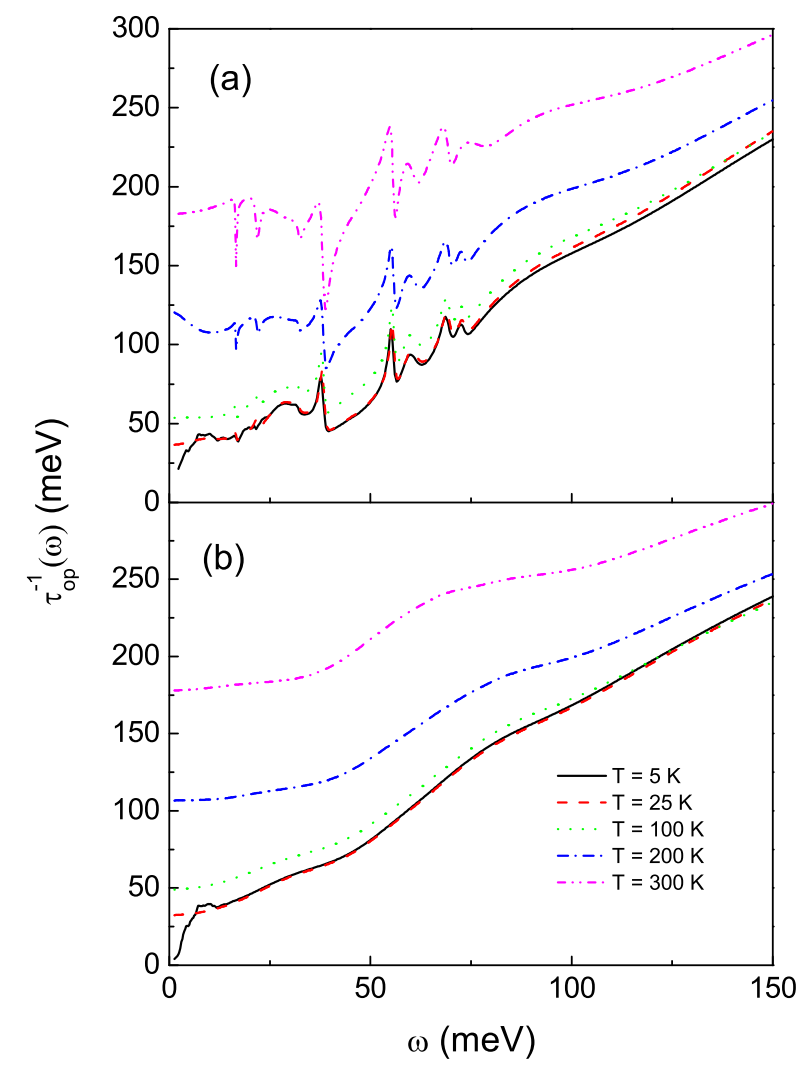

FIG. 2: (Color online) The optical scattering rate $\tau_{\text {op }}^{-1}(\omega)$ im $\mathrm{meV}$ in an optimally doped $(x=0.15)$ PCCO thin film $\left(T_{c}=21 \mathrm{~K}\right)$ for several temperatures above and below $T_{c}$ with (a) the infrared active phonon modes present and (b) with these phonons removed.

tor of two is not far from the difference in dc-conductivity $\left[\sigma_{1}(\omega)\right.$ extrapolated to $\left.\omega=0\right]$ observed in these two samples $\left(3 \times 10^{4} \Omega^{-1} \mathrm{~cm}^{-1}\right.$ for the crystal; $1.7 \times 10^{4} \Omega^{-1} \mathrm{~cm}^{-1}$ for the film).

\section{DATA ANALYSIS AND DISCUSSION}

\section{A. The $x=0.15$ PCCO single crystal}

Because of the rather large normal state zero frequency offset of the optical scattering rate PCCO can no longer be treated in the clean limit as has been done so far for the systems Bi2212, LSCO, Tl2201, and YBCO. Thus, the impurity scattering rate $\tau_{\mathrm{imp}}^{-1}$ gains importance in Eq. (4) and has to be treated as an external parameter in the maximum entropy deconvolution of this equation.

The complete inversion procedure consists of two steps. First Eq. (4) together with the appropriate kernel is deconvoluted using a classical maximum entropy method. The default model is set to a constant which is adjusted from temperature to temperature to ensure best possible 
data reproduction at high energies $\omega>250 \mathrm{meV}$. This results in a first approximation for the electron-exchange boson interaction spectral density $I^{2} \chi(\omega)$. In a second step this approximate spectral density is refined using a least squares fit procedure based on the full non-linear Eliashberg equations (A.1). This second step is of particular importance when inverting superconducting state data.

Fig. 3 reports the results of our data analysis. Figure 3(a) demonstrates the quality of data reproduction when the $I^{2} \chi(\omega)$ spectra shown in Fig. 3(b) are used to calculate the optical scattering rate using the Eliashberg equations (A.1). The heavy lines represent the data and the thin lines theory. For the normal state, $T=295 \mathrm{~K}, 200 \mathrm{~K}, 100 \mathrm{~K}$, and $30 \mathrm{~K}$ Eliashberg equation A.1b with $\tilde{\Delta}\left(\nu+i 0^{+} ; \vartheta\right) \equiv 0$ was used to calculate the renormalized quasiparticle energies $\tilde{\omega}\left(\nu+i 0^{+}\right)$. In this case the impurity scattering rate $\tau_{\text {imp }}^{-1}$ is only a term which is added to the renormalized energies. An impurity parameter $t^{+}=3.1 \mathrm{meV}$ (see Appendix ) was required for best over all data reproduction. It corresponds to an impurity scattering rate $\tau_{\mathrm{imp}}^{-1}=2 \pi t^{+} \approx 19.5 \mathrm{meV}$ for all temperatures. This is in excellent agreement with the zero frequency offset of $\sim 22 \mathrm{meV}$ reported for the $T=30 \mathrm{~K}$ data.

Particular attention is required for the superconducting state. First of all, impurities are always pair-breaking in $d$-wave superconductors and thus effectively reduce the critical temperature of the impure sample in comparison to the 'clean limit' critical temperature. Furthermore, there are two limits of impurity scattering to be considered. One limit is described by unitary or resonant scattering which is characterized by the parameter $\Gamma^{+}$in Eq. A.1b). The other limit is Born's scattering (or weak scattering) characterized by the impurity parameter $t^{+}$. In reality the scattering law is intermediate between unitary and Born limit scattering. This is characterized by the parameter $c$ in Eq. (A.1b); $c=0$ is the unitary limit and $c \rightarrow \infty$ the Born limit. Another complication arises from the fact that the relevant kernel (6) does not contain impurity scattering in contrast to its normal state counterpart Eq. (5). Furthermore, Eq. (6) requires some knowledge of the size of the gap amplitude $\Delta$. Fortunately, this is not critical in this particular case because the rather big impurity scattering rate of $\sim 22 \mathrm{meV}$, suggests that the sample will already be in the gapless regime even at $T=5 \mathrm{~K} \stackrel{35}{=}$ Thus, it will be sufficient for the first step, the maximum entropy deconvolution of Eq. (4), to use that value of $\Delta$ for which the best data reproduction can be achieved. This procedure results in a first approximation of the $I^{2} \chi(\omega)$ spectrum which is then parameterized and improved by a least squares fit to the data using the solutions of the full non-linear Eliashberg equations to calculate the optical scattering rate. In this step the impurity parameter $t^{+}$stays unchanged and only Born scattering is treated but is included in each iteration of the Eliashberg equations A.1 and, of course, this greatly reduces the value of the critical temperature $T_{c}$.

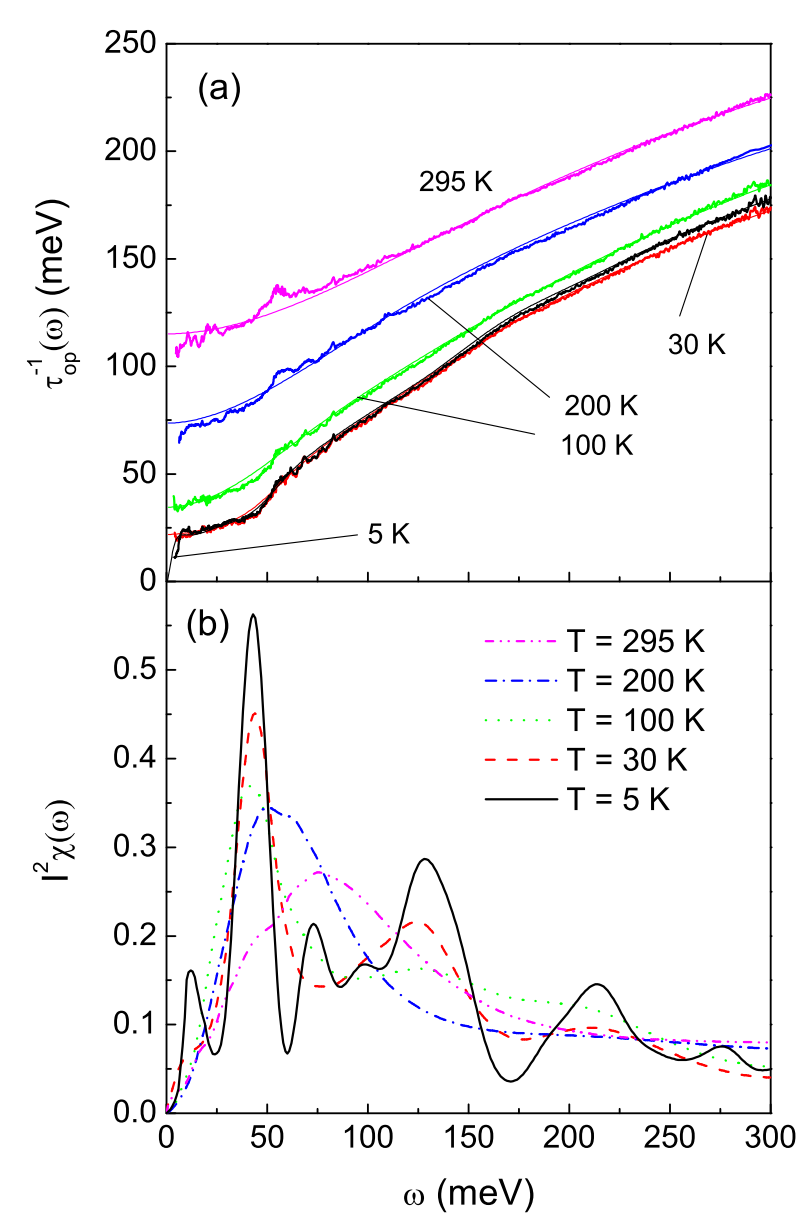

FIG. 3: (Color online) (a): The optical scattering rate $\tau_{\mathrm{op}}^{-1}(\omega)$ in $\mathrm{meV}$ as a function of $\omega$ (in $\mathrm{meV}$ ) at five temperatures, namely $T=300 \mathrm{~K}$ (magenta, dash-double dotted line), $200 \mathrm{~K}$ (blue, dash-dotted line), $100 \mathrm{~K}$ (green, dotted line), and $30 \mathrm{~K}$ (red, dashed line) in the normal state and $T=5 \mathrm{~K}$ [black solid line, the only curves with $\left.\tau_{\text {op }}^{-1}(\omega=0) \equiv 0\right]$ in the superconducting state for the optimally doped PCCO single crystal $(x=0.15)$. The heavy lines present experimental data while the light lines correspond to theoretical results obtained from solutions of the full Eliashberg equations (A.1) using the $I^{2} \chi(\omega)$ spectral densities shown in the bottom frame of this figure. The impurity parameter was set to $t^{+}=\Gamma^{+}=3.1 \mathrm{meV}$. (b): The electron-exchange boson interaction spectral density $I^{2} \chi(\omega)$ obtained from the inversion of the experimental data presented by the heavy lines in the top frame of this figure.

The result are the spectral density $I^{2} \chi(\omega)$ shown by the solid line in Fig. 3(b).

In a final step the low energy regime $0 \leq \omega \leq 10 \mathrm{meV}$ in which the initial slope of $\tau_{\mathrm{op}}^{-1}(\omega)$ is dominated by impurity scattering is to be fitted using full non-linear Eliashberg theory, Eqs. (A.1), taking into account the different laws of impurity scattering. As the scattering rate itself has already been determined, the appropriate scattering law can now be determined by the best possible fit to 


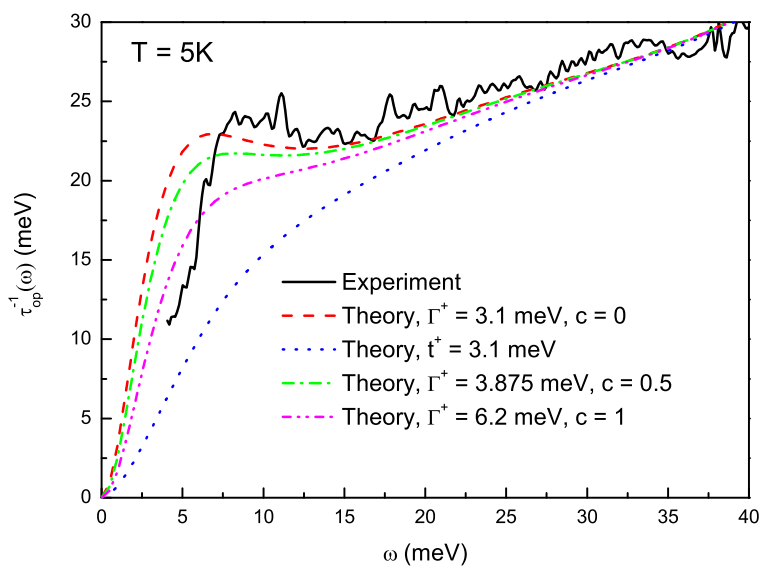

FIG. 4: (Color online) The solid black line represents the experimental $\tau_{\mathrm{op}}^{-1}(\omega)$ as a function of $\omega$ at $T=5 \mathrm{~K}$. The dashed curve presents the results of an Eliashberg theory calculation using unitary impurity scattering described by the impurity parameter $\Gamma^{+}=3.15 \mathrm{meV}$. The dotted line corresponds to Born limit scattering described by the parameter $t^{+}=3.15 \mathrm{meV}$. Finally, the dash-dotted curve represents an intermediate case with $\Gamma^{+}=3.94 \mathrm{meV}$ and $c=0.5$ and, furthermore, the dash-double dotted curve belongs to $\Gamma^{+}=6.3 \mathrm{meV}$ and $c=1$.

the data in this low energy region. Fig. 4 demonstrates the results of such a procedure. The solid black line corresponds to the experimental data, the dashed line represents unitary limit scattering with $\Gamma^{+}=3.1 \mathrm{meV}$ and the dotted line shows the result for Born limit scattering with $t^{+}=3.1 \mathrm{meV}$. For comparison we include additional results for intermediate impurity scattering, namely $\Gamma^{+}=3.875 \mathrm{meV}$ with $c=0.5$ and $\Gamma^{+}=6.2 \mathrm{meV}$ and $c=1$. Obviously, impurity scattering is much closer to unitary than to Born type scattering but it is impossible to decide whether a better fit is found for $c=0$ (unitary scattering) or $c=0.5$ (intermediate scattering). It also becomes apparent that impurity scattering does not affect the energy dependence of $\tau_{\mathrm{op}}^{-1}(\omega)$ for energies $\omega>30 \mathrm{meV}$ and, hence, there is no need to get yet a new estimate for $I^{2} \chi(\omega)$. It is certainly interesting to note in passing that the clean limit (intrinsic) critical temperature $T_{c 0}$ for $t^{+}=0$ is approximately $61 \mathrm{~K}$ so that the presence of impurity scattering has greatly reduced the value of the critical temperature.

We return to Fig. 3(b) which presents the spectral densities $I^{2} \chi(\omega)$ as a result of the inversion process. Five temperatures are shown, namely $T=5 \mathrm{~K}$ (solid line), $30 \mathrm{~K}$ (dashed line), $100 \mathrm{~K}$ (dotted line), $200 \mathrm{~K}$ (dashdotted lines), and $295 \mathrm{~K}$ (dash-double dotted line). The main feature is a very pronounced peak centered around $\sim 45 \mathrm{meV}$ and which gets reduced in amplitude with increasing temperature. This main peak is followed by a valley-hump structure which also becomes less pro-

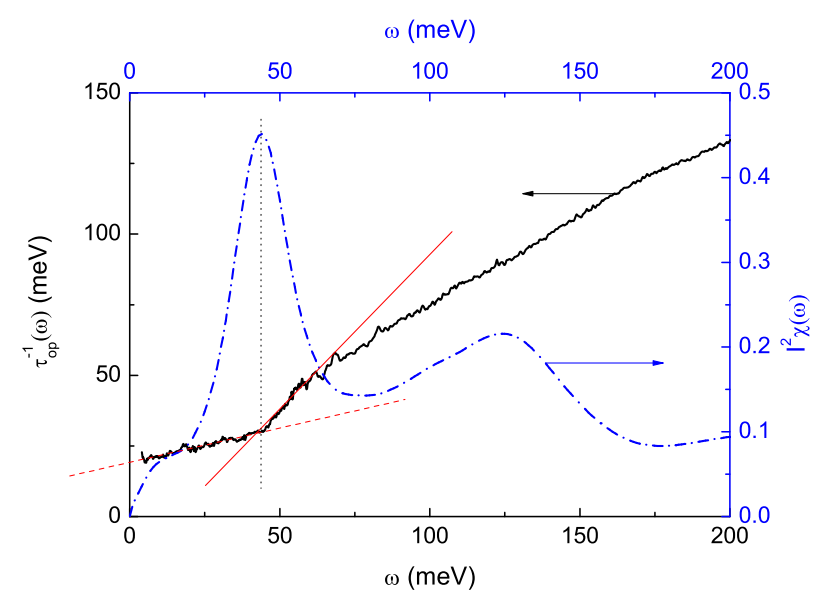

FIG. 5: (Color online) The single crystal experimental optical scattering rate $\tau_{\mathrm{op}}^{-1}(\omega)$ as a function of $\omega$ at $T=30 \mathrm{~K}$ (black solid line). The two straight lines (red, dashed and solid) are used to emphasize the change in slope in $\tau_{\mathrm{op}}^{-1}(\omega)$ at $\omega=45 \mathrm{meV}$. The (blue) dash-dotted line corresponds to the spectral density $I^{2} \chi(\omega)$ derived from the $\tau_{\mathrm{op}}^{-1}(\omega)$ data by inversion.

nounced with increasing temperature an has disappeared at $T=200 \mathrm{~K}$. At $T=295 \mathrm{~K}$ the $I^{2} \chi(\omega)$ spectral density takes on the form of a simple MMP-form as was proposed by Millis et $a l^{36}$ for a spin-fluctuation spectrum with its maximum at $\sim 80 \mathrm{meV}$. In these respects the $I^{2} \chi(\omega)$ spectral density found for the optimally doped PCCO single crystal follows the pattern found for all other hole doped High- $T_{c}$ superconductors analyzed so far. It is, finally, important to point out that the $T=5 \mathrm{~K}$ shows an additional peak centered around $\sim 12.5 \mathrm{meV}$. This peak describes a possible coupling of the charge carriers to a mode at this energy.

We add Figs. $[5$ and 6 to make two important points. Fig. 5 shows the $\tau_{\mathrm{op}}^{-1}(\omega)$ data for $T=30 \mathrm{~K}$ (solid line) for the single crystal. Superimposed are two straight lines. The first, (red) dashed line starts at the $\omega=0$ offset at the value of the residual scattering rate $\tau_{\mathrm{op}}^{-1}(\omega=0)$ and follows experiment up to about $45 \mathrm{meV}$. The second, (red) solid line starts near the origin and follows the data for energies $>45 \mathrm{meV}$ emphasizing the change in slope at $\omega=45 \mathrm{meV}$. If the first were perfectly flat (which it is not) the two straight lines would represent two processes, the Drude (coherent) part of the scattering rate and the boson assisted (incoherent) part, respectively. If for the latter we assumed that the boson is an Einstein mode at some energy $\omega_{E}$ which is large enough that the boson assisted processes are well separated from the coherent Drude part, then the coherent part of $\tau_{\mathrm{op}}^{-1}(\omega)$ would be constant and equal to the residual scattering rate at $\omega=0$ until $\omega=\omega_{E}$ is reached. At $\omega_{E}$ the boson assisted absorption sets in as an additional process and $\tau_{\mathrm{op}}^{-1}(\omega)$ develops a kink as is suggested by the intercept of the 


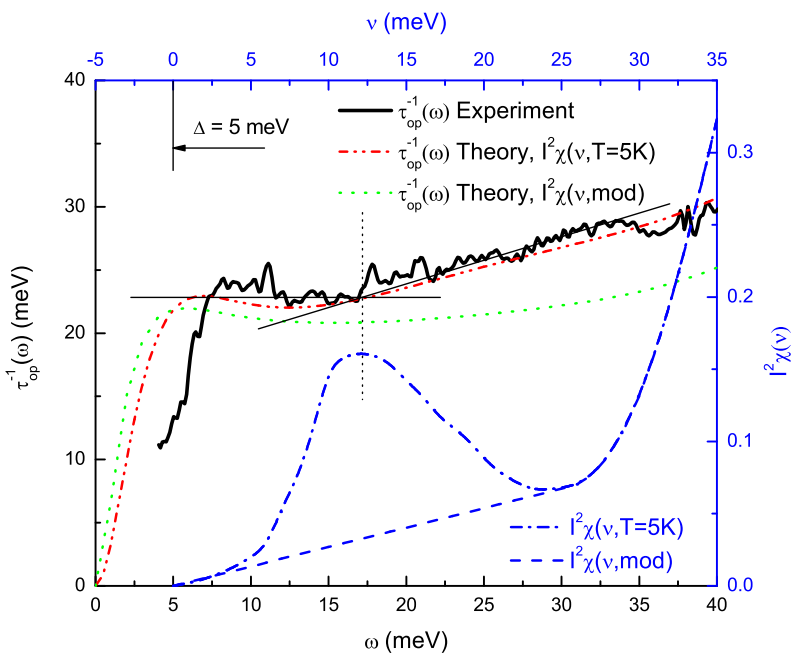

FIG. 6: (Color online) The optical scattering rate $\tau_{\mathrm{op}}^{-1}(\omega)$ vs $\omega$ for the optimally doped $(x=0.15)$ PCCO single crystal in the superconducting state at $T=5 \mathrm{~K}$. The (black) heavy solid line corresponds to experiment. The two (black) light solid lines are used to emphasize the change in slope in $\tau_{\mathrm{op}}^{-1}(\omega)$ at $\omega \approx 17 \mathrm{meV}$. The (red) dash-double dotted line represents the theoretical $\tau_{\text {op }}^{-1}(\omega)$ calculated using the spectral density $I^{2} \chi(\nu, T=5 \mathrm{~K})$ (blue, dash-dotted line) for unitary impurity scattering with $\Gamma^{+}=3.1 \mathrm{meV}$ and $c=0$. The (green) dotted line shows the theoretical $\tau_{\text {op }}^{-1}(\omega)$ calculated using the spectral density $I^{2} \chi(\nu, \bmod )$ (blue, dashed line) for the same unitary impurity scattering. The $\nu$-axis was displaced by the amplitude $\Delta=5 \mathrm{meV}$ of the superconducting gap at $T=5 \mathrm{~K}$ with respect to the $\omega$-axis.

two straight lines in Fig. 5 . This corresponds precisely to the peak at the same energy seen in the $I^{2} \chi(\omega)$ spectrum shown as a (blue) dash-dotted line in Fig. 5 with the horizontal (black) light dotted line indicating the peak center. The kink in $\tau_{\mathrm{op}}^{-1}(\omega)$ so identified is a clear signature of the peak at $45 \mathrm{meV}$ in $I^{2} \chi(\omega)$. Obviously, the (red) dashed straight line has in our case a non-zero slope as a function of energy. This is the signature of additional boson assisted processes even at energies $\omega<45 \mathrm{meV}$ and, consequently, the spectral function $I^{2} \chi(\omega)$ has non-zero weight at low energies. Next, we will examine this region more closely.

In Fig. 6] we address the question whether or not the low frequency peak at $12.5 \mathrm{meV}$ in the low temperature $I^{2} \chi(\nu, T=5 \mathrm{~K})$ spectral density (blue, dash-dotted line) is really essential for data reconstruction in the region $0 \leq \omega \leq 40 \mathrm{meV}$ and reflects features contained in the $\tau_{\text {op }}^{-1}(\omega, \bar{T}=5 \mathrm{~K})$ data. The (black) heavy solid line represents the data and, as in Fig. 5 we use two (black) thin straight lines to emphasize the change in slope which takes place at $\sim 17 \mathrm{meV}$ in $\tau_{\mathrm{op}}^{-1}(\omega, T=5 \mathrm{~K})$. For comparison we also present the spectral density $I^{2} \chi(\nu, \bmod )$ in which the peak around $12.5 \mathrm{meV}$ in spectrum $I^{2} \chi(\nu, T=$ $5 \mathrm{~K})$ has been replaced by a straight line connecting the origin with the bottom of the valley at $25 \mathrm{meV}$ (blue, dashed line). As required by theory, the $\nu$-scale was displaced by the amplitude of the superconducting gap at $T=5 \mathrm{~K}, \Delta=5 \mathrm{meV}$. Following the argument already applied in our discussion of Fig. 5 it becomes obvious that the peak at $\nu=12.5 \mathrm{meV}$ in the spectral density $I^{2} \chi(\nu, T=5 \mathrm{~K})$ coincides with the change of slope of the optical scattering rate at $\sim 17 \mathrm{meV}$ as indicated by the intersection of the two (black) thin solid lines. Thus, this peak represents, indeed, a real feature of the optical scattering rate and describes the coupling of the charge carriers to a (weak) mode at $12.5 \mathrm{meV}$.

We added two more curves to this figure. The (red) dash-double dotted curve represents the solution of the full non-linear Eliashberg equations (A.1) found for the spectral density $I^{2} \chi(\nu, T=5 \mathrm{~K})$ in the superconducting state at $T=5 \mathrm{~K}$. The impurity parameters were set to $\Gamma^{+}=3.1 \mathrm{meV}$ and $c=0$. [It is identical to the (black) thin solid line in Fig. 4(a).] We see that this result follows closely the data over the whole energy region. The second, (green) dotted curve is also for the superconducting state at $T=5 \mathrm{~K}$ but now the spectral density $I^{2} \chi(\nu, \bmod )$ has been used. The impurity parameters remain unchanged. This results falls well below experiment in the region $17 \leq \omega \leq 40 \mathrm{meV}$, thus demonstrating the importance of the $12.5 \mathrm{meV}$ peak in $I^{2} \chi(\nu, T=5 \mathrm{~K})$ for best possible data reconstruction. It is worth noting that the dashed-double dotted and the dashed curves meet only at higher energies around $70 \mathrm{meV}$. This demonstrates clearly that a peak in $I^{2} \chi(\omega)$ centered around the energy $\omega_{E}$ not only produces a kink in $\tau_{\mathrm{op}}^{-1}\left(\omega=\omega_{E}\right)$ but is also responsible for increased scattering at all energies above $\omega_{E}$.

\section{B. The $x=0.15$ thin PCCO film}

We followed the procedure outlined in the previous subsection to invert the optical scattering rate $\tau_{\mathrm{op}}^{-1}(\omega)$ of an optimally doped, epitaxially grown thin PCCO film $\left(x=0.15, T_{c}=21 \mathrm{~K}\right)$ described in Sec. IIB The best over all data reproduction of the normal state data required an impurity parameter $t^{+}=5.0 \mathrm{meV}$ which corresponds to an impurity scattering rate $\tau_{\text {imp }}^{-1}=31.4 \mathrm{meV}$ $\left(\sim 253 \mathrm{~cm}^{-1}\right)$ in excellent agreement with the experimental value of $32.5 \mathrm{meV}\left(\sim 262 \mathrm{~cm}^{-1}\right)$ at $T=25 \mathrm{~K}$. In the superconducting state the best agreement was found for an intermediate impurity scattering strength described by the parameters $\Gamma^{+}=7.8125 \mathrm{meV}$ and $c=0.75$. We also note that in this case the clean limit critical temperature $T_{c 0} \approx 57 \mathrm{~K}$. This is a remarkable result as it compares well with the intrinsic critical temperature of $61 \mathrm{~K}$ found for the single crystal. Thus, we conclude that, indeed, both samples present the same physical properties typical for optimally doped PCCO.

In discussing the results of our data analysis we primarily concentrate on the similarities in the electronboson spectral densities $I^{2} \chi(\omega)$ for the two optimally doped samples investigated here. For this purpose Fig. [7 


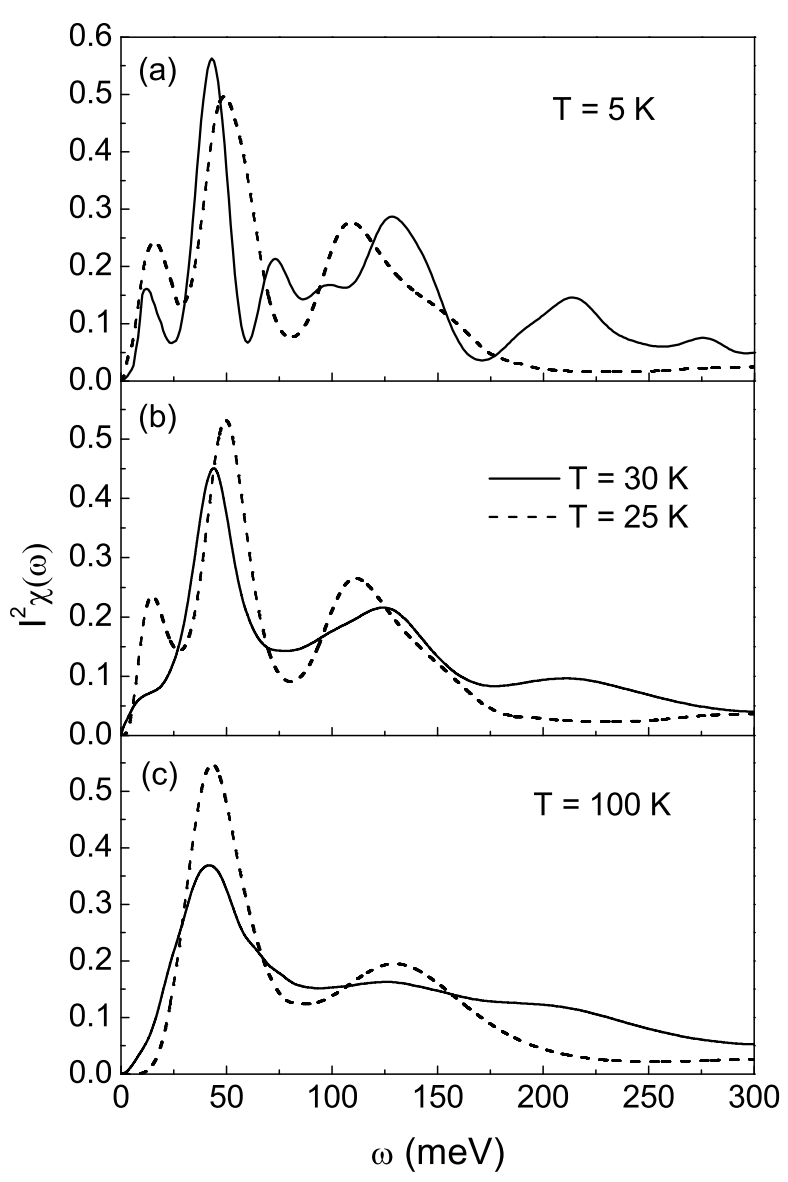

FIG. 7: The electron spectral density $I^{2} \chi(\omega)$ vs $\omega$ for the PCCO $x=0.15$ single crystal (solid lines) and thin film (dashed lines). The $I^{2} \chi(\omega)$ of the thin film has been scaled by a factor of 0.5 while the single crystal data is unchanged. This emphasizes similarities between the two sets of spectra. The results are (a) for the superconducting state at $T=5 \mathrm{~K}$, (b) for the normal state at $T=30 \mathrm{~K}$ in the single crystal is compared with the $T=25 \mathrm{~K}$ spectrum for the thin film, and (c) for the normal state at $T=100 \mathrm{~K}$.

presents the $I^{2} \chi(\omega)$ spectra for the $x=0.15$ thin film (dashed lines) scaled by a constant factor of 0.5 while the corresponding spectra of the single crystal (solid lines) stay unchanged. Figure 7 (a) is for $T=5 \mathrm{~K}$ in the superconducting state and the two spectra reveal very similar features as functions of $\omega$. There is a first resonance like peak at $\sim 14 \mathrm{meV}(\sim 12 \mathrm{meV}$ in the single crystal) which corresponds in energy to the spin one resonance reported by Wilson et $a l^{2} \stackrel{23}{\text { and }}$ to the peak at the same energy found by STM experiments 25 in the system PLCCO. There is a second, main peak at $50 \mathrm{meV}$ in the thin film sample. The single crystal $I^{2} \chi(\omega)$ spectrum shows this peak at the slightly lower energy of $\sim 45 \mathrm{meV}$. There exists, as yet, no experimental evidence from neutron scattering for the existence of a corresponding peak in the imaginary part of the spin susceptibility at such an energy in PCCO and, therefore, our analysis represents a specific prediction. This second peak is followed by a valley-hump feature which starts at $\sim 80 \mathrm{meV}$ with the hump centered at $\sim 107 \mathrm{meV}$. After this structure the spectral density levels off to a weak background which extends beyond $\omega=300 \mathrm{meV}$ and which has little structure in the case of the thin film. This result is very similar to what has been reported recently for the system LSCO by analysis of optical data 21 (including the valley-hump structure) and by neutron scattering $\underline{\underline{19}}$ It is interesting to note an additional valley-peak structure beginning at $\sim 55 \mathrm{meV}$ in the single crystal spectrum which cannot be observed at higher temperatures. This structure is most likely due to not completely removed structures in the data around this energy.

Figure 7(b) shows the single crystal results for $T=$ $30 \mathrm{~K}$ (solid line) and the thin film result for $T=25 \mathrm{~K}$ (dashed line) in the normal state; both scaled as described above. While at $T=25 \mathrm{~K}$ a small signature of the $14 \mathrm{meV}$ peak remains, it has basically vanished at $T=30 \mathrm{~K}$ in the single crystal (only a small shoulder is left). These differences could also be due to the very different nature of the two samples. The second peak is still very pronounced and stays basically unchanged in energy in both samples. The valley-hump structure which follows this second peak at around $80 \mathrm{meV}$ stays unchanged in comparison to the $T=5 \mathrm{~K}$ spectra as is the background for $\omega>200 \mathrm{meV}$.

Finally, Fig. 7(c) presents the rescaled spectra for $T=100 \mathrm{~K}$. We see that all structures have been smeared out in the spectra and the positions of the main peak change only little. The valley-hump structure which follows the main peak has been smeared out almost completely in the $I^{2} \chi(\omega)$ spectral density of the single crystal while it is still well developed in the thin film spectral density. It is also remarkable that the thin film spectra have a largely suppressed background for $\omega>180 \mathrm{meV}$ in comparison to the single crystal spectra. Increasing the temperature further results in spectra which are MMPform ${ }^{36}$ like and the maximum at $T=300 \mathrm{~K}$ moves to $\sim 80 \mathrm{meV}$ in the thin film and to $\sim 90 \mathrm{meV}$ in the single crystal. This corresponds to what has been observed in optimally doped Bi2212 $\underline{18}$

We also note that the maximum of the hump in the $T=5 \mathrm{~K}$ and $25 \mathrm{~K}$ thin film spectra is at lower energies as compared to the same structure in the single crystal spectra. At $100 \mathrm{~K}$, though, they agree, i.e.: in the $x=0.15$ thin film spectra the maximum in the high energy valley-hump structure moves towards higher energies with increasing temperatures while it stays fixed in the single crystal.

\section{SUMMARY}

Motivated by the report of a spin one resonance in the imaginary part of the spin susceptibility in the electron-doped system PLCCO at the very low energy of 
$\sim 11 \mathrm{meV}$ by neutron scattering we studied the optical scattering rate reported for optimally doped PCCO samples using the maximum entropy technique to extract information on the electron-boson spectral density $I^{2} \chi(\omega)$. This spectral density contains information on the coupling of the charge carriers to bosonic modes. We found that in the superconducting state the electrons couple to a bosonic mode centered around $12(14) \mathrm{meV}$ and that there is a second higher energy group of modes centered around 45(50) meV which has not yet been observed by neutron scattering. Above $T_{c}$ the thin film sample still shows some coupling to the low energy mode at $25 \mathrm{~K}$ and while in the single crystal no coupling to this mode can be observed at $T=30 \mathrm{~K}$ which can certainly be due to the differences in the two samples. The second, high energy peak is clearly developed above $T_{c}$ in all samples and evolves into an MMP-form like spin fluctuation background with further increasing temperature. All $I^{2} \chi(\omega)$ spectra extend to energies $>300 \mathrm{meV}$. These results resemble very closely to what has been observed in optimally doped LSCO samples and, apart from the low energy mode, what has been reported for optimally Bi2212 samples. All this proves that the electron-doped system PCCO behaves in its charge dynamics much like all the other hole-doped High- $T_{c}$ cuprates. There is one important difference though, PCCO in contrast to all hole-doped High- $T_{c}$ superconductors investigated so far, is in the dirty limit. The residual scattering in all samples investigated here is sufficiently large to substantially reduce the value of the critical temperature over its pure (intrinsic) limit. This fact is in full agreement with results reported by Dagan et al $\stackrel{37}{5}$ from their analysis of PCCO-lead tunneling junctions.

\section{Acknowledgment}

Research supported in part by the Natural Sciences and Engineering Research Council of Canada (NSERC) and by the Canadian Institute for Advanced Research (CIFAR). We thank N. Bontemps for valuable discussions and her keen interest. JPC and ES want to thank T. Timusk for his interest and many discussions. ES enjoyed the hospitality and friendship of the members of the Department of Physics and Astronomy during his visit at McMaster University. The Work at Brookhaven National Laboratory was supported by the Office of Science, U.S. Department of Energy, under Contract No. DE-AC02-98CH10886.

\section{APPENDIX: $d$-WAVE ELIASHBERG EQUATIONS FOR IMPURE SYSTEMS}

The generalization to a $d$-wave gap has already been published by Jiang et al. $\frac{38}{3}$ and has been used to describe various aspects of the superconducting state in the cuprates. In the mixed representation of Marsiglio et $a l^{39}$ they are of the form

$$
\begin{aligned}
\tilde{\Delta}\left(\nu+i 0^{+} ; \vartheta\right)= & \pi T g \sum_{m=0}^{\infty} \cos (2 \vartheta)\left[\lambda\left(\nu-i \omega_{m}\right)+\lambda\left(\nu+i \omega_{m}\right)\right] h_{+}\left(i \omega_{m}\right) \\
& +i \pi g \int_{-\infty}^{\infty} d z \cos (2 \vartheta) I^{2} \chi(z)[n(z)+f(z-\nu)] h_{-}\left(i \omega_{m} \rightarrow \nu-z+i 0^{+}\right),
\end{aligned}
$$

and, in the renormalization channel,

$$
\begin{aligned}
\tilde{\omega}\left(\nu+i 0^{+}\right)= & \nu+i \pi T \sum_{m=0}^{\infty}\left[\lambda\left(\nu-i \omega_{m}\right)-\lambda\left(\nu+i \omega_{m}\right)\right] g_{+}\left(i \omega_{m}\right) \\
& +i \pi \int_{-\infty}^{\infty} d z I^{2} \chi(z)[n(z)+f(z-\nu)] g_{-}\left(i \omega_{m} \rightarrow \nu-z+i 0^{+}\right) \\
& +i \pi \Gamma^{+} \frac{g_{-}\left(i \omega_{n} \rightarrow \nu+i 0^{+}\right)}{c^{2}+g_{-}^{2}\left(i \omega_{n} \rightarrow \nu+i 0^{+}\right)+h_{-}^{2}\left(i \omega_{n} \rightarrow \nu+i 0^{+}\right)} .
\end{aligned}
$$

Here

$$
h_{ \pm}\left(i \omega_{m}\right)=\left\langle\frac{\tilde{\Delta}\left(i \omega_{m} ; \vartheta\right) \cos (2 \vartheta)}{\sqrt{\tilde{\omega}^{2}\left(i \omega_{m}\right) \pm \tilde{\Delta}^{2}\left(i \omega_{m} ; \vartheta\right)}}\right\rangle_{\vartheta}, \quad g_{ \pm}\left(i \omega_{m}\right)=\left\langle\frac{\tilde{\omega}\left(i \omega_{m}\right)}{\sqrt{\tilde{\omega}^{2}\left(i \omega_{m}\right) \pm \tilde{\Delta}^{2}\left(i \omega_{m} ; \vartheta\right)}}\right\rangle_{\vartheta}
$$

and the parameter $g$ in Eq. A.1a allows for a possible difference in spectral density between the $\tilde{\omega}$ and $\tilde{\Delta}$ 
critical temperature. In the above $\tilde{\Delta}\left(i \omega_{m} ; \vartheta\right)$ is the pairing energy which its evaluated at the fermionic Matsubara frequencies $\omega_{m}=\pi T(2 m-1), m=0, \pm 1, \pm 2, \ldots$ and $\tilde{\omega}\left(i \omega_{m}\right)$ are the renormalized frequencies evaluated at the same Matsubara frequencies; $f(z)$ and $n(z)$ are the Fermi and Bose distribution, respectively. Furthermore, the $\vartheta$ dependence of the pairing energy is described by $\tilde{\Delta}\left(i \omega_{m}, \vartheta\right)=\tilde{\Delta}\left(i \omega_{m}\right) \cos (2 \vartheta)$ with $\vartheta$ the polar angle in the two-dimensional $\mathrm{CuO}$ Brillouin zone. The brackets $\langle\cdots\rangle_{\vartheta}$ are the angular average over $\vartheta$, and $\lambda(\nu)=\int_{-\infty}^{\infty} d \Omega \alpha^{2} F(\Omega) /\left(\nu-\Omega+i 0^{+}\right)$. Eqs. (A.1) are a set of nonlinear coupled equations for the renormalized pairing potential $\tilde{\Delta}\left(\nu+i 0^{+} ; \vartheta\right)$ and the normalized frequencies $\tilde{\omega}\left(\nu+i 0^{+}\right)$with the gap $\Delta\left(\nu+i 0^{+} ; \vartheta\right)=\tilde{\Delta}(\nu+$ $\left.i 0^{+} ; \vartheta\right) / Z(\nu)$, where the renormalization function $Z(\nu)$ was introduced in the usual way as $\tilde{\omega}\left(\nu+i 0^{+}\right)=\nu Z(\nu)$. Finally, $\tilde{\Delta}\left(i \omega_{n}, \vartheta\right)$ and $\tilde{\omega}\left(i \omega_{n}\right)$ are the solutions of the equivalent equations formulated on the imaginary axis. $\underline{40}$

Impurity scattering is described by the term proportional to $\Gamma^{+}$in Eq. A.1b and enters only this equation because we assume a pure $d$-wave model for the pairing potential with zero average over the Fermi surface while the impurity scattering is assumed to be isotropic. Here, $\Gamma^{+}$is proportional to the impurity concentration and $c$ is related to the electron phase shift for scattering off the impurity. For unitary or resonant scattering $c$ is equal to zero while $c \rightarrow \infty$ gives the Born approximation, i.e.: the weak scattering limit. In this limit the entire impurity term reduces to the form $i \pi t^{+} g\left(i \omega_{n} \rightarrow \nu+i 0^{+}\right)$ with $c$ absorbed into the impurity parameter $t^{+}$. In the normal state $\tilde{\Delta}\left(\nu+i 0^{+} ; \vartheta\right) \equiv 0$ and there is no need to distinguish any longer between unitary and Born limit impurity scattering. The scattering term reduces to $i \pi t^{+} \operatorname{sgn}\left[\tilde{\omega}\left(i \omega_{n} \rightarrow \nu+i 0^{+}\right)\right]$. At the critical temperature linearized Eliashberg equations are valid, i.e.: $h\left(i \omega_{n}\right) \simeq 0$ and $g\left(i \omega_{n}\right) \simeq \operatorname{sgn}\left[\tilde{\omega}\left(i \omega_{n}\right)\right]$. Thus, at $T_{c}$, $t^{+}\left\{c^{2}+\operatorname{sgn}\left[\tilde{\omega}\left(i \omega_{n}\right)\right]\right\}=\Gamma^{+}$and this relates immediately $\Gamma^{+}$to the impurity scattering rate via $\tau_{\text {imp }}^{-1}=2 \pi t^{+}$.

The optical conductivity follows from knowledge of $\tilde{\omega}$ and $\tilde{\Delta}$. The formula to be evaluated is

$$
\sigma_{o p}(T, \nu)=\frac{\Omega_{p}^{2}}{4 \pi} \frac{i}{\nu}\left\langle\int_{0}^{\infty} d \omega \tanh \left(\frac{\beta \omega}{2}\right)[J(\omega, \nu)-J(-\omega, \nu)]\right\rangle_{\vartheta} .
$$

The function $J(\omega, \nu)$ is given by

$$
2 J(\omega, \nu)=\frac{1-N(\omega ; \vartheta) N(\omega+\nu ; \vartheta)-P(\omega ; \vartheta) P(\omega+\nu ; \vartheta)}{E(\omega ; \vartheta)+E(\omega+\nu ; \vartheta)}+\frac{1+N^{*}(\omega ; \vartheta) N(\omega+\nu ; \vartheta)+P^{*}(\omega ; \vartheta) P(\omega+\nu ; \vartheta)}{E^{*}(\omega ; \vartheta)-E(\omega+\nu ; \vartheta)},
$$

with $E(\omega ; \vartheta)=\sqrt{\tilde{\omega}^{2}\left(\omega+i 0^{+}\right)-\tilde{\Delta}^{2}\left(\omega+i 0^{+} ; \vartheta\right)}$, $N(\omega ; \vartheta)=\tilde{\omega}\left(\omega+i 0^{+}\right) / E(\omega ; \vartheta)$, and $P(\omega ; \vartheta)=$
$\tilde{\Delta}\left(\omega+i 0^{+} ; \vartheta\right) / E(\omega ; \vartheta)$. Finally, the star refers to the complex conjugate.
* Electronic address: schachinger@itp.tu-graz.ac.at

1 F. Marsiglio, T. Startseva, and J. P. Carbotte, Physics Lett. A 245, 172 (1998).

2 J. P. Carbotte, E. Schachinger, and D. Basov, Nature (London) 401, 354 (1999).

${ }^{3}$ P. Bourges, Y. Sidis, H. F. Fong, B. Keimer, L. P. Regnault, J. Bossy, A. S. Ivanov, D. L. Lilius, and I. Aksay, in High Temperature Superconductivity, edited by S. E. Barnes, A. Ashkenazi, J. L. Cohn, and F. Zuo (American Institute of Physics, Woodbury, New York, 1999), p. 207.

${ }^{4}$ P. Dai, H. A. Mook, S. M. Hayden, G. Aeppli, T. G. Perring, R. D. Hunt, and F.Doğan, Science 284, 1344 (1999).

5 E. Schachinger, J. P. Carbotte, and D. Basov, Europhys. Lett. 54, 380 (2001).

6 E. Schachinger and J. Carbotte, Phys. Rev. B 62, 9054 (2000).

7 H. He, P. Bourges, Y. Sidis, C. Ulrich, L. Regnault,
S. Pailhès, N. S. Berzigiarova, N. N. Kolesnikov, and B. Keimer, Science 295, 1045 (2002).

8 S. V. Dordevic, C. C. Homes, J. J. Tu, T. Valla, M. Strongin, P. D. Johnson, G. D. Gu, and D. Basov, Phys. Rev. B 71, 104529 (2005).

${ }^{9}$ S. V. Shulga, O. V. Dolgov, and E. G. Maksimov, Physica C 178, 266 (1991).

10 J. P. Cabotte and E. Schachinger, Ann. Phys. (Leipzig) 15, 585 (2006).

11 P. B. Allen, Phys. Rev. B 3, 305 (1971).

12 A. Abanov, A. V. Chubukov, and J. Schmalian, Phys. Rev. B 63, 180510(R) (2001).

13 E. Schachinger and J. Carbotte, Phys. Rev. B 64, 094501 (2001).

14 J. J. Tu, C. C. Homes, G. D. Gu, D. Basov, and M. Strongin, Phys. Rev. B 66, 144514 (2002).

15 E. Schachinger, D. Neuber, and J. P. Carbotte, Phys. Rev. B 73, 184507 (2006). 
16 E. T. Jaynes, in Maximum Entropy and Bayesian Methods, edited by J. Skilling (Kluwer, Dordrecht, 1989).

17 See for instance: D.S . Sivia, Data Analysis (Clarendon Press, Oxford 1996), pp. 119.

18 J. Hwang, T. Timusk, E. Schachinger, and J. Carbotte, Phys. Rev. B 75, 144508 (2007).

19 B. Vignolle, S. M. Hayden, D. F. McMorrow, H. M. Rønnow, B. Lake, C. D. Frost, and T. Perring, Nature Physics 3, 148 (2007).

${ }^{20}$ F. Gao, D. B. Romero, D. B. Tanner, J. Talvacchio, and M. G. Forrester, Phys. Rev. B 47, 1036 (1993).

21 J. Hwang, E. Schachinger, J. P. Carbotte, F. Gao, D. Tanner, and T. Timusk, Phys. Rev. Lett. 100, 137005 (2008).

22 X. J. Zhou, J. Shi, T. Yoshida, T. Cuk, W. L. Yang, V. Bruet, J. Nakamura, N. Manella, S. Komiya, Y. Ando, et al., Phys. Rev. Lett. 95, 117001 (2005).

23 S. D. Wilson, P. Dai, S. Li, S. Chi, H. J. Kang, and J. W. Lynn, Nature (London) 442, 59 (2006).

24 M. Fujita, M. Matsuda, S.-H. Lee, M. Nakagawa, and K. Yamada, Phys. Rev. Lett. 101, 107003 (2008).

25 F. C. Niestemski, S. Kunwar, S. Zhou, S. Li, H. Ding, Z. Wang, P. Dai, and V. Madhavan, Nature (London) 450, 1058 (2007).

26 C. C. Homes, R. P. S. M. Lobo, P. Fournier, A. Zimmers, and R. L. Greene, Phys. Rev. B 74, 214515 (2006).

27 A. Zimmers, R. P. S. M. Lobo, N. Bontemps, C. C. Homes, M. C. Barr, Y. Dagan, and R. L. Greene, Phys. Rev. B 70, 132502 (2004).

28 A. Zimmers, J. M. Tomczak, R. P. S. M. Lobo, N. Bontemps, C. P. Hill, M. C. Barr, Y. Dagan, R. L. Greene, A. J. Millis, and C. C. Homes, Europhys. Lett. 70, 225
(2005).

29 D. Y. Smith, in Handbook of Optical Constants of Solids, edited by E. D. Palik (Academic, New York, 1985), pp. 35-68.

30 J. Hwang, T. Timusk, A. V. Puchkov, N. L. Wang, G. D. $\mathrm{Gu}$, C. C. Homes, J. J. Tu, and H. Eisaki, Phys. Rev. B 69, 094520 (2004).

31 C. C. Homes, A. W. McConnell, B. P. Clayman, D. A. Bonn, R. Liang, W. N. Hardy, M. Inoue, H. Negishi, P. Fournier, and R. L. Greene, Phys. Rev. Lett. 84, 5391 (2000).

32 C. C. Homes, Q. Li, P. Fournier, and R. L. Greene, Phys. Rev. B 66, 144511 (2002).

33 M. Braden, L. Pintschovius, T. Uefuji, and K. Yamada, Phys. Rev. B 72, 184517 (2005).

34 A. F. Santander-Syro, R. P. S. M. Lobo, N. Bontemps, W. Lopera, D. Girata, Z. Konstantinovic, Z. Z. Li, and H. Raffy, Phys. Rev. B 70, 134504 (2004).

35 A. A. Abrikosov and L. Gor'kov, Sov. Phys. JETP 12, 1243 (1961).

36 A. Millis, H. Monien, and D. Pines, Phys. Rev. B 46, 14803 (1990).

37 Y. Dagan, R. Beck, and R. Greene, Phys. Rev. Lett. 99, 147004 (2007).

38 C. Jiang, E. Schachinger, J. P. Carbotte, D. Basov, and T. Timusk, Phys. Rev. B 54, 1264 (1996).

39 F. Marsiglio, M. Schossmann, and J. P. Carbotte, Phys. Rev. B 37, 4965 (1988).

40 E. Schachinger, J. P. Carbotte, and F. Marsiglio, Phys. Rev. B 56, 2738 (1997). 